\title{
Focus on the problem of carbapenem resistance in clinical significant non- fermenters: Acinetobacter baumannii and Pseudomonas aeruginosa
}

\author{
Petrova $A^{1,2^{*}}$, Murdjeva $\mathbf{M}^{1,2}$ \\ ${ }^{1}$ Department of Microbiology and Immunology, Faculty of Pharmacy, Medical University-Plovdiv, Bulgaria \\ ${ }^{2}$ Laboratory of Microbiology, University Hospital “St. George”-Plovdiv, Bulgaria
}

Accepted on December 13, 2017

\section{Editorial}

Few decades after the implementation of the potential broad spectrum antimicrobials, the antibiotic resistance turns into a global problem. The non-fermenters of clinical significance Acinetobacter baumannii and Pseudomonas aeruginosa, usually considered as opportunistic pathogens, nowadays emerge to evolve in ones of the most problematic causative agents of infections. The therapeutic restrictions are determined on one hand due to their intrinsic resistance to a great variety of antibiotics and on the other to the arising acquired resistance. The carbapenem resistance is a growing tendency worldwide and usually it is not an isolated phenomenon, but combined with resistance against other antibiotic groups. Carbapenems not long ago assumed to be, "the heavy artillery" of the antibiotic therapy today face the disability to be effective in the treatment of infections caused by multi-drug-resistant bacteria (MDR). The production of bla OXA-23, OXA-24, OXA-58 and the hyperexpression of OXA-51 due to ISAba1 insertion sequence are the leading cause of carbapenem resistance in Acinetobacter baumannii [1]. This tendency is observed in Bulgaria as well [2-5]. The loss of OprD transmembrane protein sometimes combined with overexpression of the intrinsic AmpC and the overexpression of some efflux pumps are considered as main factors for carbapenem resistance in Pseudomonas aeruginosa whereas metallo-enzymes' production has a secondary role [6,7]. Two major studies proved that in Bulgarian clinical isolates Pseudomonas aeruginosa the increased expression or overexpression of MexXY-OprM efflux pump sometimes combined with overexpression of MexAB-OprM with or without loss of the OprD is the main mechanism of determining this type of resistance. Metallo-enzyme production and overexpression of the intrinsic AmpC are rarely involved [8,9].

Both methods-phenotypic and genetic, are used to evaluate the mechanisms of resistance. As phenotypic tests for detection of common carbapenemases usually a Modified Hodge test and a Carba NP-test with its variations are applied [10-12]. Both methods are cheap, easily performed and with reliable results and good correlation with the results obtained from the molecular-genetic screening.

For the screening of metallo-beta-lactamases double-disk combined test with disks imipenem $(10 \mu \mathrm{g})$ and imipenem/ EDTA $(10 / 760 \mu \mathrm{g})$ [13] and combined E-test with imipenem $(4-256 \mu \mathrm{g} / \mathrm{mL})$ and imipenem $(1-64 \mu \mathrm{g} / \mathrm{mL})+$ constant level of EDTA [14] are methods of choice.
A classical PCR investigation is used mainly for scientific research to detect the most often carbapenemases for both microorganisms and a qRT-PCR to assess the expression of genes encoding the proteins of leading efflux pumps as MexAB-OprM and MexXY-OprM, the intrinsic AmpC and the transmembrane receptor OprD in Pseudomonas. Subsequently RAPD analysis or any other phylogenetic analysis is recommendatory to be performed for determination of clonal affiliation between strains

Studying the exact structure of antimicrobial resistance is a cornerstone in coping with imposed therapeutic restriction. On the ground of numerous research results new strategies are elaborated. They include the use of sulbactam (with or without ampicillin or cephoperazone) in the treatment of infections provoked by OXA-23 producing A.baumannii due to the lack of activity of these carbapenemases against it and the intrinsic effect of this beta-lactamase inhibitor against Acinetobacter spp. Combinations including colistin, rifampicin and fosphomycin could be an appropriate alternative for infections provoked by both Pseudomonas and Acinetobacter [15-17]. On the other hand the newly available in Europe antimicrobial combinations ceftazidime-avibactam and ceftolozanetazobactam show good results in managing infections caused by carbapenem resistant Pseudomonas which are not MBL producers, but exhibit increased efflux or/and decreased influx [18]. Several efflux pumps inhibitors were reported as potential antimicrobials which could be implemented in the treatment of carbapenem resistant Pseudomonas due to increased efflux in future [19]. New techniques try to turn antibiotics targeted for Gram-positive bacteria only due to their incapacity to cross the outer membrane in potential antimicrobials for Gram-negative as well. Through a novel drug delivery system: wrapping molecules into fusogenic liposomes, vancomycin could be implemented in the treatment of multi-drug-resistant nonfermenters [20].

"Prophylaxis is the best treatment". The most powerful weapon against multi-drug-resistant microorganisms is to manage to stop their emerging. This aim could be accomplished if mutant prevention concentrations (MPC) are imposed in the problematic clinical units. Prescriptions of levofloxacin 750 $\mathrm{mg} / 24 \mathrm{~h}+$ imipenem $1 \mathrm{gr} / 12 \mathrm{~h}$ tend to prevent the emerge of carbapenem resistant $P$. aeruginosa and to eradicate the existing isolates with hyperexpression of MexAB-OprM and MexXY-OprM efflux pumps even with loss of the transmembrane receptor OprD [21]. Not at last place the strict epidemiological and hygiene control. Patients with proved infections of MDR microorganisms should be isolated and treated with precaution. The hospital staff as a main source of 
Citation: Petrova A, Murdjeva M. Focus on the problem of carbapenem resistance in clinical significant non-fermenters: Acinetobacter baumannii and Pseudomonas aeruginosa Microbiol Curr Res. 2018;2(1):3-4.

spreading MDR microbes should undergo regular educational trainings on prevention and managing such kind of infections. Finally good hospital politics is mandatory: buying only the cheapest antibiotics could have a negative economic effect as well as saving budget at the expense of a reduction of the personnel.

\section{References}

1. Poirel L, Nordmann P. Carbapenem resistance in Acinetobacter baumannii: Mechanisms and epidemiology. Clin Microb Infec. 2006;12:826-36

2. Stoeva T, Higgins PG, Savov E et al. Nosocomial spread of OXA-23 and OXA-58 beta-lactamase-producing Acinetobacter baumannii in a Bulgarian hospital. J Antimicrob Chemother. 2009;63:618-20.

3. Strateva T, Markova B, Marteva-Proevska $\mathrm{Y}$ et al. Widespread dissemination of multidrug-resistant Acinetobacter baumannii producing OXA-23 carbapenemase and ArmA 16S ribosomal RNA methylase in a Bulgarian UNIVERSITY hospital. Brazilian J Infect Dis. 2012;16:307-10

4. Petrova A, Stanimirova I, Ivanov I et al. Carbapenemase production of clinical isolates Acinetobacter baumannii and Pseudomonas aeruginosa from a Bulgarian University Hospital. Folia medica. 2017;59.

5. Todorova B, Velinov T, Ivanov I et al. First detection of OXA-24 carbapenemase-producing Acinetobacter baumannii isolates in Bulgaria. World $\mathrm{J}$ Microbiol Biotechnol. 2014;30:1427-30.

6. Livermore DM. Pseudomonas, porins, pumps and carbapenems. J Antimicrob Chemother. 2001;47:247-50

7. Quale J, Bratu S, Gupta J, Landman D. Interplay of efflux system, ampC and oprD expression in carbapenem resistance of Pseudomonas aeruginosa clinical isolates. Antimicrob Agents Chemother. 2006;50:1633-41

8. Vatcheva-Dobrevska R, Mulet X, Ivanov I et al. Molecular Epidemiology and Multidrug Resistance Mechanisms of Pseudomonas aeruginosa Isolates from Bulgarian Hospitals. Microb Drug Resist. 2013;19:355-61

9. Petrova A, Borisova T, Feodorova Y et al. Analysis of OprD receptor in carbapenem resistant clinical isolates Pseudomonas aeruginosa and interplay between the expression of main efflux pumps and intrinsic AmpC. C. R. Acad. Bulg. Sci.

10. Lee K, Chong Y, Shin HB et al. Modified Hodge and EDTA-disk synergy tests to screen metallo- $\beta$-lactamaseproducing strains of Pseudomonas and Acinetobacter species. Clin Microbiol. 2001;7:88-91.

11. Dortet L, Poirel L, Errera C et al. CarbAcineto NP test for rapid detection of carbapenemase- producing Acinetobacter sp. J Clin Microbiol. 2014;52:2359-64.

12. Dortet L, Poirel L, Nordmann P. Rapid detection of carbapenemase-producing Pseudomonas spp. J Clin Microbiol. 2012;50:3773-6.

13. Picão RC, Andrade SS, Nicoletti AG et al. Metallo- $\beta$ lactamase detection: Comparative evaluation of double- disk synergy versus combined disk tests for IMP, GIM, SIM, SPM, or VIM producing isolates. J Clin Microbiol. 2008;46:2028-37.

14. Berges L, Rodriguez-Villalobos H, Deplano A et al. Prospective evaluation of imipenem/EDTA combined disc and Etest for detection of metallo-beta-lactamaseproducing Pseudomonas aeruginosa . Journal of Antimicrobial Chemotherapy. 2007;59:812-3.

15. Aydemir H, Akduman D, Piskin N et al. Colistin vs. the combination of colistin and rifampicin for the treatment of carbapenem-resistant Acinetobacter baumannii ventilatorassociated pneumonia. Epidemiol Infect . 2013;141:1214 22.

16. Batirel A, Balkan II, Karabay $\mathrm{O}$ et al. Comparison of colistin-carbapenem, colistin-sulbactam, and colistin plus other antibacterial agents for the treatment of extremely drug-resistant Acinetobacter baumannii bloodstream infections. Eur J Clin Microbiol Infect Dis. 2014;33:131122.

17. Santimaleeworagun W, Wongpoowarak $\mathrm{P}$, Chayakul $\mathrm{P}$ et al. In vitro activity of colistin or sulbactam in combination with fosfomycin or imipenem against clinical isolates of carbapenem-resistant acinetobacter baumannii producing OXA-23 carbapenemases. Southeast Asian J Trop Med Public Health. 2011;42:890-900.

18. Van Duin D, Bonomo RA. Ceftazidime/Avibactam and Ceftolozane/Tazobactam: Second-generation $\beta$-Lactam $/ \beta$ Lactamase Inhibitor Combinations. Clin Infect Dis. 2016;63:234-41.

19. Cortez-Cordova J, Kumar A. Activity of the efflux pump inhibitor phenylalanine-arginine $\beta$-naphthylamide against the AdeFGH pump of Acinetobacter baumannii. Int $\mathrm{J}$ Antimicrob Agents. 2011;37:420-4.

20. Nicolosi D, Scalia M, Nicolosi VM et al. Encapsulation in fusogenic liposomes broadens the spectrum of action of vancomycin against Gram-negative bacteria. Int J Antimicrob Agents. 2010;35:553-8.

21. Lister PD, Wolter DJ, Wickman PA et al. Levofloxacin/ imipenem prevents the emergence of high-level resistance among Pseudomonas aeruginosa strains already lacking susceptibility to one or both drugs. J Antimicrob Chemother. 2006;57:999-1003

\section{*Corresponding author}

Atanaska Petrova

Department of Microbiology and Immunology,

Faculty of Pharmacy,

Medical University-Plovdiv,

Bulgaria

Tel: 00359888016628

E-mail: atanasia_petroff@abv.bg 